\title{
Foot Osteoarthritis: Addressing an Overlooked Global Public Health Problem
}

Yvonne M. Golightly, PT, PhD, 1,2,3,4 and Lucy S. Gates, PhD, 5,6

${ }^{1}$ Department of Epidemiology, University of North Carolina at Chapel Hill, Chapel Hill, North Carolina, USA

${ }^{2}$ Thurston Arthritis Research Center, University of North Carolina at Chapel Hill, Chapel Hill, North Carolina, USA

${ }^{3}$ Injury Prevention Research Center, University of North Carolina at Chapel Hill, Chapel Hill, North Carolina, USA

${ }^{4}$ Division of Physical Therapy, University of North Carolina at Chapel Hill, Chapel Hill, North Carolina, USA

${ }^{5}$ Centre for Sport, Exercise and Osteoarthritis Research Versus Arthritis, University of Southampton, Southampton, UK

${ }^{6}$ Nuffield Department of Orthopaedics, Rheumatology and Musculoskeletal Sciences, Botnar Research Centre, University of Oxford, Oxford, UK

Corresponding Author:

Yvonne M. Golightly, PT, PhD

Thurston Arthritis Research Center

University of North Carolina

3300 Thurston Building, Campus Box 7280

Chapel Hill, NC 27599-7280

Email: golight@email.unc.edu

\section{Key words}

Foot, osteoarthritis, phenotypes, epidemiology, study design

Word Count $=1,486$, Pages $=5$, References $=12$

Tables/Figures $=0$

(C) 2020, The Authors. Arthritis Care \& Research published by Wiley Periodicals, Inc. on behalf of American College of Rheumatology.

This is an open access article under the terms of the Creative Commons Attribution License, which permits use, distribution and reproduction in any medium, provided the original work is properly cited. 


\section{Author contributions}

Both Dr. Golightly and Dr. Gates contributed to the conception and design of this report, drafted and revised the manuscript, and provided final approval of the submitted version.

\section{Conflict of interest statement}

The authors have no competing interests or financial disclosures in relation to this work.

(C) 2020, The Authors. Arthritis Care \& Research published by Wiley Periodicals, Inc. on behalf of American College of Rheumatology. 
Foot pain affects at least one in three adults over the age of 45 (1), and one in six adults $\geq 50$ years old with foot pain also have radiographic evidence of foot osteoarthritis (OA) (2). Unfortunately, our knowledge of foot OA and its burden substantially lags behind that of other joint sites (i.e., knee, hip, and hand). Given the link of foot pain and disorders with disability and reduced quality of life (3), a greater understanding is needed of foot OA and its pathogenesis and risk factors, especially since it is likely a serious disease with a significant public health burden.

In this issue, Arnold et al. (4) advance the field of foot OA with their novel study on associations of foot and leg muscle strength with symptomatic midfoot OA, a frequent type of foot $\mathrm{OA}$. This study builds on prior research demonstrating that associations of muscle weakness with OA at the hip, knee, and hand and with pain and impaired physical function. In this cross-sectional study, 52 participants with midfoot OA demonstrated less strength in all foot and leg muscle groups (i.e., ankle plantarflexors, dorsiflexors, invertors, and evertors; hallux and lesser digit plantarflexors) compared to 36 asymptomatic individuals without radiographic midfoot OA. Additionally, greater ankle invertor strength was associated with less foot pain among individuals with midfoot OA. Based on the study design, the direction of these associations is not known, but these results provide initial data to support a future investigation of foot and leg muscle strengthening as a potential treatment for symptomatic midfoot OA.

The authors call attention to limitations of their study, including criteria for defining absence of midfoot OA and a smaller sample size of the asymptomatic participant group. They also acknowledge the necessity for larger samples and longitudinal studies to examine the role of foot and leg muscle weakness with pain and structural outcomes in the midfoot, along with a need to investigate foot and leg muscle strengthening as a treatment for midfoot OA. These significant research needs, along with limitations observed in other studies of foot OA, suggest multiple research areas required for progressing our understanding of the etiology of foot OA and its management. The purpose of this editorial is to discuss important considerations for improving future foot $O A$ research across populations globally. Establishing standard definitions and measures of foot $O A$ is essential for longitudinal analyses to identify risk factors for and subgroups of foot OA, as well as for clinical trials of treatments for this highly prevalent and disabling condition.

(C) 2020, The Authors. Arthritis Care \& Research published by Wiley Periodicals, Inc. on behalf of American College of Rheumatology. 
The current knowledge gaps in foot OA are in part due to a lack of longitudinal population based data to determine the number of people who may require care and to estimate the burden of foot OA among the general population. For the foot, there is a lack of agreed clinical definitions for many measures; for example, there is currently no agreement on how to capture OA-related foot pain. Only a handful of international cohorts collect foot data, but unlike the knee and hip, measures of $\mathrm{OA}$, pain, and physical function often are not comparable across cohorts, therefore limiting comparative estimates across geographical regions.

Previously, defining radiographic foot OA was limited by the Kellgren-Lawrence grading system, which depended greatly on the presence of osteophytes. This scoring system may not be reliable at the foot, where joint space narrowing could occur alone or prior to osteophyte formation. Until recently, previous studies have focused mainly on first metatarsophalangeal (MTP) joint radiographic $\mathrm{OA}$, likely due to difficulties with radiographic interpretation at the smaller more complex joints. An important advancement in the field of foot OA was the development of the La Trobe Foot Atlas (5), which individually scores radiographic presentation of osteophytes and joint space narrowing at the first MTP joint and four midfoot joints: first and second cuneo-metatarsal joints, navicular- first cuneiform joint, and talo-navicular joint. This atlas, which was used by Arnold et al. (4), has improved our ability to estimate the prevalence of foot OA in populations. With the recent emergence of longitudinal radiographic foot data, questions have arisen over how to define incident and progressive disease, and unfortunately, the La Trobe Foot Atlas was not designed to address these issues. For example, would we consider a foot joint with OA to have a clinically important worsening of OA if there is an increase solely in the osteophyte score, or should scores of both the osteophyte and joint space narrowing grades progress? Do these features have equal weighting at each of the five joints? Should radiographic OA in one joint in the foot be considered radiographic foot $\mathrm{OA}$, or should this definition depend on multiple joint involvement? If the presence of radiographic features is required in multiple joints, how many and which joints?

Evidence for phenotypes of foot OA has begun to emerge, with three distinct classes of radiographic foot OA: no/minimal foot OA, isolated first MTP joint OA, and polyarticular foot OA (6).

(C) 2020, The Authors. Arthritis Care \& Research published by Wiley Periodicals, Inc. on behalf of American College of Rheumatology. 
This work is a useful start in the understanding of patterns of involvement across different joints in the feet, but further investigation across large cohorts is needed.

Questions over the radiographic definition of incidence and progression previously have arisen at the knee and the hand, with solutions suggested based on years of previously published work in the field (7-10). Because of the potential multiple joint involvement in foot OA, definitions used for incidence and progression of hand OA may serve as a model $(8,9)$. Perhaps, the definition of radiographic foot OA would vary based on which joint or joints are involved and their potential association with pain and disability, in which case we should consider which features correspond to such clinical factors.

Defining symptomatic OA at the foot is necessary for producing estimates that are meaningful to clinicians and patients. To date, large cross-sectional cohort studies of symptomatic foot OA have been limited by general definitions of foot pain (e.g., presence of pain anywhere in the foot) $(2,11)$. A notable strength of the study by Arnold et al. was the use of a foot manikin and palpation of midfoot joints to determine that foot pain was present in the same region as the joints of interest, providing an example of how other studies could define symptomatic OA in a particular region of the foot. Definitions of foot pain also should consider the duration, quality (e.g., sharp, ache, stiffness), and severity of symptoms. Defining worsening of symptomatic foot OA is considerably more complicated than radiographic foot OA. For instance, would a worsening of symptoms in the presence of stable radiographic features at the foot be considered worsening of symptomatic OA, or should radiographic features progress as well? Likewise, would worsening in structural severity of either or both features in the presence of stable symptoms be considered worsening symptomatic OA?

Other possible ways of defining foot OA that could be developed include the use of images other than radiography, such as magnetic resonance imaging and ultrasound. A clinical definition that could be broadly used to help clinicians diagnose foot OA without imaging would be valuable. Similar to the American College of Rheumatology Diagnostic Guidelines for knee, hip and hand OA, a clinical definition of foot OA could be developed based on patient age and the presence of signs and symptoms.

(C) 2020, The Authors. Arthritis Care \& Research published by Wiley Periodicals, Inc. on behalf of American College of Rheumatology. 
Due to a lack of longitudinal investigation and consistent definitions of foot outcomes, there is little evidence available regarding the potential risk factors for foot OA, without which it is difficult to develop interventional research. In fact, there are few randomized controlled trials in foot OA. Existing trials provide initial evidence for the effectiveness of pain relief of physical therapy, rocker sole shoes, foot orthoses and surgical interventions in first MTP joint OA and prefabricated orthoses in midfoot OA (12). The role of occupational activities, injuries, and physical activity in foot OA are not known; longitudinal investigations of these factors would determine their part in disabling foot $\mathrm{OA}$ and inform interventions.

In summary, standardizing definitions of foot OA would help us better understand the disease pathogenesis and risk factors, perhaps delineating different phenotypes of foot OA that require distinctive management approaches. By ensuring that future work related to foot OA can be compared across the globe, the true burden of foot OA can be established. As quality longitudinal data are gathered over time, we can gain a better comprehension of the natural history, treatment response, and economic impact of foot OA. Lessons learned for defining incident and progressive OA at the knee and hand can be applied to the foot. With standard definitions, clinical trials could be implemented to determine the effectiveness of applying existing OA management approaches to foot OA or developing new interventions. We are at a pivotal time for providing definitions that can be used in all future longitudinal studies, which will inform the global public health impact of foot OA and advance our ability to treat this disabling condition.

(C) 2020, The Authors. Arthritis Care \& Research published by Wiley Periodicals, Inc. on behalf of American College of Rheumatology. 


\section{References}

1. Golightly YM, Hannan MT, Shi XA, Helmick CG, Renner JB, Jordan JM. Association of foot symptoms with self-reported and performance-based measures of physical function:

The Johnston County osteoarthritis project. Arthritis Care Res (Hoboken). 2011;63(5):654-9.

2. Roddy E, Thomas MJ, Marshall M, Rathod T, Myers H, Menz HB, et al. The population prevalence of symptomatic radiographic foot osteoarthritis in community-dwelling older adults: cross-sectional findings from the clinical assessment study of the foot. Ann Rheum Dis. 2015;74(1):156-63.

3. Thomas MJ, Roddy E, Zhang W, Menz HB, Hannan MT, Peat GM. The population prevalence of foot and ankle pain in middle and old age: a systematic review. Pain. $2011 ; 152(12): 2870-80$.

4. Arnold JB, Halstead J, Grainger AJ, Keenan AM, Hill CL, Redmond AC. Foot and leg muscle weakness in people with midfoot osteoarthritis. Arthritis Care Res (Hoboken). In press.

5. Menz HB, Munteanu SE, Landorf KB, Zammit GV, Cicuttini FM. Radiographic classification of osteoarthritis in commonly affected joints of the foot. Osteoarthritis Cartilage. 2007;15(11):1333-8.

6. Rathod T, Marshall M, Thomas MJ, Menz HB, Myers HL, Thomas E, et al. Investigations of Potential Phenotypes of Foot Osteoarthritis: Cross-Sectional Analysis From the Clinical Assessment Study of the Foot. Arthritis Care Res (Hoboken). 2016;68(2):217-27.

(C) 2020, The Authors. Arthritis Care \& Research published by Wiley Periodicals, Inc. on behalf of American College of Rheumatology. 
7. Felson DT, Niu J, Guermazi A, Sack B, Aliabadi P. Defining radiographic incidence and progression of knee osteoarthritis: suggested modifications of the Kellgren and Lawrence scale. Ann Rheum Dis. 2011;70(11):1884-6.

8. Haugen IK, Englund M, Aliabadi P, Niu J, Clancy M, Kvien TK, et al. Prevalence, incidence and progression of hand osteoarthritis in the general population: the Framingham Osteoarthritis Study. Ann Rheum Dis. 2011;70(9):1581-6.

9. Haugen IK, Magnusson K, Turkiewicz A, Englund M. The Prevalence, Incidence, and Progression of Hand Osteoarthritis in Relation to Body Mass Index, Smoking, and Alcohol Consumption. J Rheumatol. 2017;44(9):1402-9.

10. Zhang Y, Niu J, Felson DT, Choi HK, Nevitt M, Neogi T. Methodologic challenges in studying risk factors for progression of knee osteoarthritis. Arthritis Care Res (Hoboken). 2010;62(11):1527-32.

11. Flowers P, Nelson AE, Hillstrom HJ, Renner JB, Jordan JM, Golightly YM. Cross-sectional analysis of foot osteoarthritis frequency and associated factors: The Johnston County Osteoarthritis Project. Arthritis Rheumatol. 2017;69(S10).

12. Roddy E, Menz HB. Foot osteoarthritis: latest evidence and developments. Ther Adv Musculoskelet Dis. 2018;10(4):91-103.

(C) 2020, The Authors. Arthritis Care \& Research published by Wiley Periodicals, Inc. on behalf of American College of Rheumatology. 


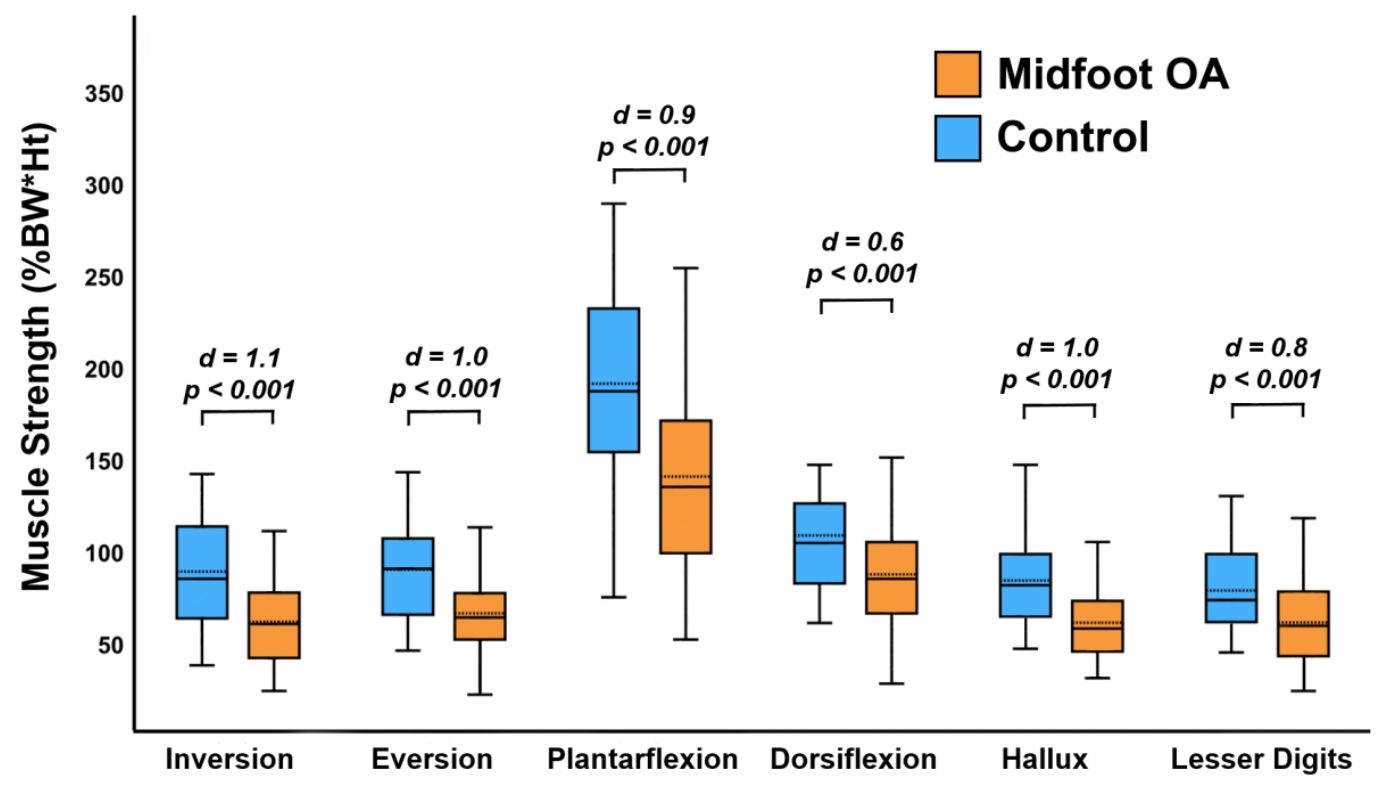

Figure 1. Muscle strength for foot and leg muscle groups for midfoot OA and control participants. Boxplots represent median and interquartile range. Dashed lines indicate mean value with corresponding effect size (d) and $p$-value for differences in means between groups for each variable.

(C) 2020, The Authors. Arthritis Care \& Research published by Wiley Periodicals, Inc. on behalf of American College of Rheumatology. 


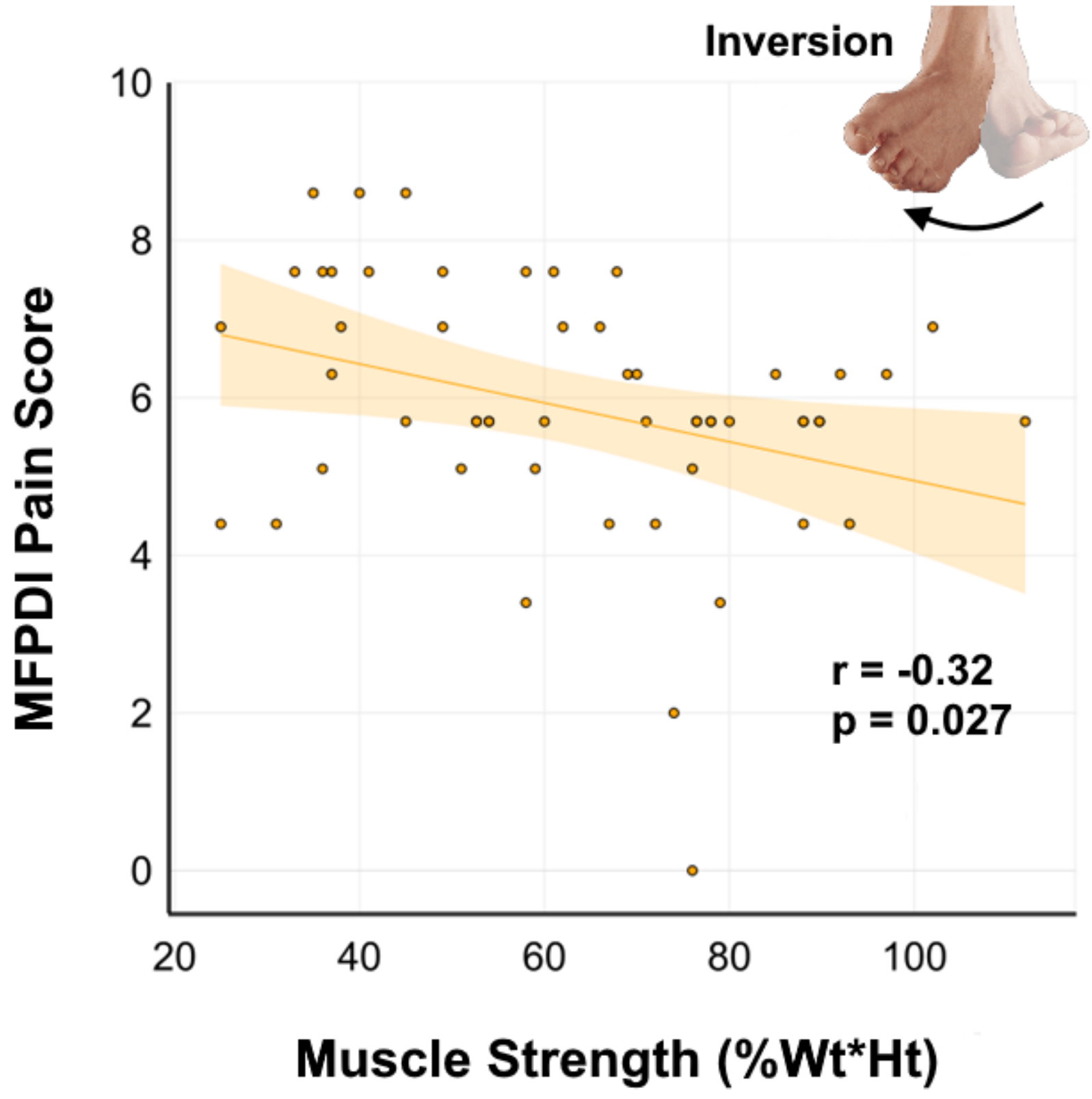

Figure 2. Relationship between invertor muscle strength and MFPDI pain in people with midfoot osteoarthritis

(C) 2020, The Authors. Arthritis Care \& Research published by Wiley Periodicals, Inc. on behalf of American College of Rheumatology. 